International Journal of Canadian Studies

Revue internationale d'études canadiennes

\title{
Contribution au développement socioéconomique de la région d'Edmonton par les femmes africaines noires francophones immigrées entre 2000 et 2006
}

\author{
Ismaëlie Hyppolite
}

Numéro 45-46, 2012

Francophonies, Interculturality, Cultures and Strategies

Francophonies, interculturalité, cultures et stratégies

URI : https://id.erudit.org/iderudit/1009905ar

DOI : https://doi.org/10.7202/1009905ar

Aller au sommaire du numéro

\section{Éditeur(s)}

Conseil international d'études canadiennes

\section{ISSN}

1180-3991 (imprimé)

1923-5291 (numérique)

\section{Découvrir la revue}

\section{Citer cet article}

Hyppolite, I. (2012). Contribution au développement socioéconomique de la région d'Edmonton par les femmes africaines noires francophones immigrées entre 2000 et 2006. International Journal of Canadian Studies / Revue internationale d'études canadiennes, (45-46), 239-259.

https://doi.org/10.7202/1009905ar

\section{Résumé de l'article}

Ce travail concerne les femmes africaines francophones installées à Edmonton depuis au moins cinq ans. En s'installant dans une nouvelle société, les nouveaux arrivants font face à des normes établies et dépendamment de la situation et de l'environnement, les systèmes subissent des modifications. Ces changements ont des conséquences sur la vie des immigrants et également sur celle des membres de la communauté d'accueil. Souvent ces modifications conduisent à la différenciation, la discrimination, l'inégalité et l'exclusion. Ces phénomènes vécus particulièrement par les immigrants, peuvent influencer leur contribution socioéconomique au développement de la région d'établissement. Au cours des entretiens et des focus group réalisés avec les femmes africaines francophones faisant partie de notre étude, nous avons eu l'occasion de mettre en évidence non seulement des éléments favorables à leur intégration mais aussi certains éléments qui sont considérés comme des barrières freinant leur effort pour arriver à une intégration socio-économique réussie. Ainsi, nous avons exploré leur niveau d'éducation, leur formation professionnelle et leurs expériences de travail à Edmonton et également aux pays d'origine. Pour que l'intégration socioéconomique se concrétise, il faut que la personne immigrante ait les mêmes possibilités qu'un membre de la communauté d'accueil d'occuper un emploi et de gagner autant que lui, si les compétences sont comparables (Centre d'études ethniques de l'Université de Montréal, 1994, p. 45). Tel n'est pas le cas pour certaines femmes qui ont participé à notre étude. Elles doivent lutter non seulement pour s'accommoder sur le plan social, mais aussi et surtout, sur le plan économique. À partir des résultats de notre recherche nous pouvons confirmer que l'intégration socioéconomique des femmes immigrantes africaines francophones n'est pas simplement une question de choix personnel, mais ce choix doit s'inscrire dans des conditions favorisant le processus d'intégration en question. 


\title{
Ismaëlie Hyppolite
}

\section{Contribution au développement socioéconomique de la région d'Edmonton par les femmes africaines noires francophones immigrées entre 2000 et 2006}

\section{Résumé}

Ce travail concerne les femmes africaines francophones installées à Edmonton depuis au moins cinq ans. En s'installant dans une nouvelle société, les nouveaux arrivants font face à des normes établies et dépendamment de la situation et de l'environnement, les systèmes subissent des modifications. Ces changements ont des conséquences sur la vie des immigrants et également sur celle des membres de la communauté d'accueil. Souvent ces modifications conduisent à la différenciation, la discrimination, l'inégalité et l'exclusion. Ces phénomènes vécus particulièrement par les immigrants, peuvent influencer leur contribution socioéconomique au développement de la région d'établissement. Au cours des entretiens et des focus group réalisés avec les femmes africaines francophones faisant partie de notre étude, nous avons eu l'occasion de mettre en évidence non seulement des éléments favorables à leur intégration mais aussi certains éléments qui sont considérés comme des barrières freinant leur effort pour arriver à une intégration socio-économique réussie. Ainsi, nous avons exploré leur niveau d'éducation, leur formation professionnelle et leurs expériences de travail à Edmonton et également aux pays d'origine. Pour que l'intégration socioéconomique se concrétise, il faut que la personne immigrante ait les mêmes possibilités qu'un membre de la communauté d'accueil d'occuper un emploi et de gagner autant que lui, si les compétences sont comparables (Centre d'études ethniques de l'Université de Montréal, 1994, p. 45). Tel n'est pas le cas pour certaines femmes qui ont participé à notre étude. Elles doivent lutter non seulement pour s'accommoder sur le plan social, mais aussi et surtout, sur le plan économique. À partir des résultats de notre recherche nous pouvons confirmer que l'intégration socioéconomique des femmes immigrantes africaines francophones n'est pas simplement une question de choix personnel, mais ce choix doit s'inscrire dans des conditions favorisant le processus d'intégration en question.

\begin{abstract}
This work concerns French-speaking African women who are settled in Edmonton for at least five years. By settling in a new society, they face established norms and depending on the situation and the environment, systems undergo modifications. These changes have consequences on the lives of immigrants and on members of the host community. Often these modifications lead to differentiation, discrimination, inequality, and exclusion. These phenomena, experienced especially by immigrants, can influence their socioeconomic
\end{abstract}


contribution to the development of the region in which they've settled. During exchanges and focus groups conducted with the French-speaking African women who participated in our study, we had the opportunity to highlight not only elements which favour their integration, but also certain elements that are considered barriers and which halt their efforts toward successful socioeconomic integration. We have thus explored their level of education, their professional training, and their work experiences in Edmonton and in their country of origin. In order for socioeconomic integration to concretize, the immigrant person must have the same opportunities as a member of the host community for obtaining employment and equal income if their skills are comparable (Centre d'études ethniques de l'Université de Montréal, 1994, $p$. 45). This was not the case for some women who participated in our study. They must battle to adapt socially but also, and mainly, economically. Based on the results of our research, we can confirm that socioeconomic integration of French-speaking African immigrant women is not simply an issue of personal choice, but this choice must be framed by conditions that favour the process of integration.

Cet article s'inscrit dans le cadre d'une réflexion sur l'immigration des femmes africaines francophones à Edmonton. Son objectif est de mettre en relief les difficultés rencontrées par ces femmes dans leur processus d'intégration, ainsi que la nature de leur contribution tant sur le plan social qu'économique. L'immigration, phénomène très compliqué, a généralement de sérieuses répercussions sur la vie de l'immigrant et particulièrement celle de la femme immigrée. Les changements vécus conduisent souvent à la différenciation sociale, à la discrimination, à l'inégalité et à l'exclusion. De plus, sur le plan identitaire, l'insertion culturelle en pays d'accueil peut entraîner chez les migrants des profondes transformations. Ils doivent, entre autres, faire face à des différences socioéconomiques et politiques. Pierre Bourdieu explique :

Qu'à l'exception des sociétés les moins différenciées, toutes les sociétés se présentent comme des espaces sociaux, c'est-à-dire des structures de différences que l'on ne peut comprendre véritablement qu'à condition de construire le principe générateur qui fonde ces différences dans l'objectivité. Ce principe est considéré comme la structure de la distribution des formes de pouvoir qui sont efficientes dans l'univers social considéré et qui varient selon les lieux et les moments. (Bourdieu 54)

Cependant, cette structure changeante peut constituer soit un champ de forces, soit un champ de lutte où les agents s'affrontent avec des moyens et des fins différenciés selon leur position dans la structure du champ de forces, contribuant ainsi à conserver ou à transformer la structure (ibid. 55). Dans cette perspective, comment les femmes africaines francophones immigrées à Edmonton peuvent-elles contribuer au développement socioéconomique de la 
région? Cette question est selon nous très pertinente, car bien que plusieurs chercheurs aient déjà travaillé sur le problème de l'immigration des femmes au Canada, aucune étude n'a été réalisée sur les femmes africaines francophones immigrées à Edmonton, notamment en ce qui a trait à leur contribution au développement socioéconomique de la région. Au cours de cette recherche, nous avons passé en revue certains éléments tels que le niveau de scolarité, la formation professionnelle, les expériences de travail acquises à Edmonton et dans les pays d'origine des femmes participantes.

Pour recueillir les informations nécessaires à notre travail, nous avons utilisé trois techniques de recherche qualitative, à savoir : la revue de la littérature, le focus group et l'entretien par questionnaire. Notre échantillonnage est constitué d'une trentaine femmes venant de différents pays de l'Afrique francophone, âgées entre 25 et 50 ans et vivant à Edmonton depuis au moins cinq ans. Pour identifier nos répondantes et respecter leur anonymat, nous avons utilisé des numéros quand il fallait rapporter des passages de leur entretien. Les numéros vont de 1 à 30 . Le tableau suivant montre le nombre de personnes interviewées par pays et leur provenance.

Choix des répondantes pour les entretien

\begin{tabular}{|c|c|c|c|}
\hline Pays d'origine & Nombre de femmes/pays & Pays d'origine & Nombre de femmes/pays \\
\hline R. D. du Congo & 6 & Côte d'Ivoire & 1 \\
\hline Rwanda & 5 & Tchad & 1 \\
\hline Algérie & 3 & Burkina Faso & 1 \\
\hline Maroc & 3 & Madagascar & 1 \\
\hline Burundi & 3 & Île Maurice & 1 \\
\hline Guinée & 2 & Sénégal & \\
\hline Cameroun & 2 & & \\
\hline
\end{tabular}

Les phénomènes qui nous intéressent dans ce travail sont l'immigration, les minorités visibles, l'emploi, l'intégration et la différentiation sociales. Cette dernière est perçue comme le fait que la société, selon ses valeurs et ses critères, donne aux individus une place prédéterminée dans l'échelle sociale, ce qui a des conséquences énormes dans leur vie tout en engendrant des inégalités. Ces phénomènes ne peuvent pas se développer à partir d'une seule et unique variable. Ils se développent à partir des relations sociales multiples et diverses, et sur la façon dont elles fonctionnent pour structurer soit le marché du travail, soit pour créer des inégalités sociales (Juteau 2003). Quoiqu'indépendants les uns des autres, ces phénomènes sont interreliés. Nous pouvons par exemple dire que le phénomène de minorité visible découle de celui de l'immigration et que l'intégration est une conséquence de l'immigration. Il nous semble important de noter que les phénomènes 
retenus pour la présente analyse caractérisent non seulement notre population cible, mais nous permettent également de comprendre que l'immigration est un phénomène très complexe qui apporte des changements considérables dans la vie des immigrants. Parmi ces derniers, le plus souvent, les femmes sont considérées comme celles qui connaissent le plus les difficultés de l'immigration. La façon dont elles s'y prennent pour réagir à ces obstacles nous a particulièrement intéressée, puisque nous avons pu à la fois découvrir et comprendre les stratégies utilisées par les femmes africaines francophones pour contribuer au développement socioéconomique de la région d'Edmonton.

\section{Immigration, phénomène compliqué}

Chaque pays a ses traditions, sa législation et ses principes spécifiques en matière d'immigration. Néanmoins, les défis qui s'imposent aux grands pays dans ce domaine sont comparables ou même identiques dans la plupart des cas. Ces pays déploient des efforts pour gérer au mieux une immigration diversifiée dans un contexte de mobilité accrue des personnes. L'immigration n'est pas un phénomène récent : les mouvements de populations ont toujours existé et les causes de ces déplacements sont multiples. Celles-ci sont d'ordre professionnel, familial, personnel, sécuritaire, politique et économique. À ces causes traditionnelles, qui provoquent généralement des flux migratoires importants, s'ajoutent les catastrophes naturelles qui deviennent de plus en plus fréquentes avec les changements climatiques. Ces dernières constituent une particularité de notre époque et provoquent de nombreux déplacements supplémentaires.

Le Canada, terre d'accueil du flux migratoire, est considéré comme l'un des pays qui ont été les plus affectés par les migrations internationales. Il faut remonter dans le temps pour dénicher les traces de cette tradition d'accueil. Avec l'arrivée des Loyalistes dans le dernier quart du XVIII siècle, le Canada a connu son premier mouvement d'immigration vraiment important. Les Loyalistes fuyaient vers le Canada après la signature du traité de Versailles en 1783 qui mettait fin à la Guerre d'indépendance. Entre 1783 et 1812, environ 30000 Loyalistes se sont établis au Canada (Couture et Bergeron 149). Depuis, malgré les contraintes et les discriminations, le nombre d'immigrants ne cesse d'augmenter. Jacques Henripin (216) rapporte qu'au cours du XX siècle, l'immigration nette a ajouté 7,3 millions de personnes à la population canadienne, sur une croissance globale de 25,3 millions.

La tendance à immigrer au Canada n'a cessé de s'amplifier ces dernières années. Selon le recensement de 2006 réalisé par Statistique Canada, on a dénombré au Canada « 6186950 personnes nées à l'étranger, soit près du cinquième $(19,8 \%)$ de la population totale du pays et la plus forte proportion enregistrée en 75 ans » (Statistique Canada, 2006). Le même organisme mentionne qu'entre 2001 et 2006, la population du Canada née à l'étranger a crû de 13,6 \%. Il s'agit d'un taux de croissance quatre fois plus élevé que 
celui de la population née au pays, lequel s'établit à 3,3\% au cours de la même période. Le recensement estime à 1110000 le nombre d'immigrants qui se sont établis au Canada entre le 1 ${ }^{\text {er }}$ janvier 2001 et le 16 mai 2006. Ces nouveaux arrivants représentent $17,9 \%$ de l'ensemble de la population née à l'étranger et 3,6\% de la population totale de 31,2 millions d'habitants au Canada. Par ailleurs, en 2006, 10,6 \% des nouveaux arrivants au Canada étaient nés en Afrique, en légère hausse également par rapport à 2001 (8,3\%), toujours selon les estimations de Statistique Canada.

\section{L'immigration des femmes au Canada et en Alberta}

Depuis toujours, les femmes ont représenté une partie importante des mouvements migratoires internationaux. À cet égard, le Canada suit la tendance et les données du recensement de 2006 démontrant que la présence des femmes immigrantes n'est pas du tout négligeable, puisqu'elle peut parfois atteindre plus de $50 \%$ de la population migrante. Dans « certains groupes (anglais, américains, antillais) le nombre de femmes excède les effectifs masculins ", relate Aleyda Lamotte (3). Malgré cette proportion considérable, la question de l'immigration féminine n'a pas toujours suscité de grands intérêts tant dans les milieux gouvernementaux que dans les milieux universitaires. Ce manque de visibilité des femmes immigrées tient au fait qu'elles ont été majoritairement admises dans le cadre de l'immigration familiale, "volet du mouvement migratoire international lui-même très peu visible, tant à l'échelle canadienne que provinciale » (ibid.). Cette vision limitative de la population féminine immigrante fut longtemps véhiculée par une recherche de type traditionnel s'intéressant essentiellement à l'ensemble de l'immigration et aux travailleurs sélectionnés. Cette approche globalisante « centrait l'analyse sur les immigrants et leur famille en postulant que les hommes sont des immigrants et leur famille, des femmes et des enfants à charge » (Lamotte 3 ).

Parmi les différents groupes récemment immigrés en Alberta, nous trouvons des femmes africaines francophones qui, elles aussi, forment un groupe d'immigrants très important. Cependant, malgré nos recherches, nous ne pouvons pas les dénombrer en raison du fait qu'aucun chiffre n'est disponible. Celles qui choisissent de s'installer à Edmonton retiennent particulièrement notre attention. Ainsi, pour mener à bien cette étude, nous nous sommes informée au sujet des recherches déjà effectuées auprès des groupes francophones s'établissant à Edmonton. Nous avons constaté que, jusqu'à présent, aucune étude particulière n'a été menée auprès des femmes africaines francophones vivant dans la région. Nous pensons qu'en étudiant leur parcours, en tant que groupe non homogène et sous l'angle de leur intégration socioéconomique, nous parviendrons à comprendre et à mettre en relief la façon dont ces femmes africaines francophones contribuent au développement socioéconomique de la région. Le tableau suivant montre la provenance et le nombre d'immigrants francophones africains arrivés en Alberta entre 2001 et 2006. 
Alberta : Immigrants francophones africains reçus entre 2001 et 2006

\begin{tabular}{|c|c|c|c|c|c|c|c|}
\hline $\begin{array}{c}\text { Pays } \\
\text { d'origine }\end{array}$ & $\mathbf{2 0 0 1}$ & $\mathbf{2 0 0 6}$ & $\begin{array}{c}\mathbf{1 9 9 1 -} \\
\mathbf{2 0 0 6}\end{array}$ & $\begin{array}{c}\text { Pays } \\
\text { d'origine }\end{array}$ & $\mathbf{2 0 0 1}$ & $\mathbf{2 0 0 6}$ & $\begin{array}{c}\mathbf{1 9 9 1 -} \\
\mathbf{2 0 0 6}\end{array}$ \\
\hline R.D.Congo & 220 & 320 & 905 & Guinée & 15 & 75 & 125 \\
\hline Rwanda & 60 & 175 & 305 & $\begin{array}{c}\text { Côte } \\
\text { d'Ivoire }\end{array}$ & 40 & 45 & 85 \\
\hline Algérie & 110 & 155 & 370 & Mauritanie & 0 & 30 & 40 \\
\hline Maroc & 80 & 105 & 455 & Tchad & 45 & 30 & 90 \\
\hline Burundi & 20 & 85 & 155 & Bénin & 0 & 30 & 30 \\
\hline Île Maurice & 75 & 80 & 425 & Madagascar & 25 & 10 & 50 \\
\hline
\end{tabular}

Source : Statistique Canada, Recensement de la population de 2006, Produit $\mathrm{n}^{\circ}$ 97557-XCB2006007 au catalogue de Statistique Canada.

Comme mentionné plus haut, notre étude porte particulièrement sur les femmes africaines francophones immigrées au Canada et qui choisissent Edmonton comme terre d'accueil. Edmonton, située dans la province de l'Alberta, en est également la capitale. Après Calgary, elle est la deuxième plus grande ville de la province. Selon Statistique Canada, la population de cette ville s'élevait à 730712 habitants en 2006, tandis que celle de la province de l'Alberta était estimée à 3290350 habitants. Entre 2001 et 2006, la population immigrante vivant à Edmonton était évaluée à 29555 habitants, dont 15520 femmes (Statistique Canada, 2007), soit $52.51 \%$.

\section{Éléments influençant l'apport des femmes africaines francophones au développement de la région d'Edmonton}

Malgré les dissemblances criantes qui existent entre les différentes catégories de femmes, il est clair qu'elles partagent une même réalité en tant que femmes. La discrimination, l'exclusion, la différenciation sociale, pour ne citer que ces difficultés, sont présentes dans leurs vies. Évoluant, bien sûr, dans des contextes socioéconomique et politique différents, les femmes éprouvent certains besoins similaires qu'il est nécessaire de combler. À ces éléments communs s'ajoutent des éléments particuliers aux femmes immigrantes qui, habituellement, vivent dans des conditions précaires et sont souvent victimes de différenciations, de discrimination et de traitements producteurs d'inégalité sociale au cours de leur intégration. Dans le contexte familial africain francophone, les femmes immigrées se sentent différenciées non seulement à cause de la langue qui constitue une barrière à leur intégration, mais aussi à cause du type d'emploi qu'elles effectuent (Mulatris 2009). Elles sont souvent surqualifiées pour des postes qu'elles arrivent difficilement, pourtant, à obtenir. De plus, leur participation à des activités socioculturelles organisées par la société d'accueil est très limitée. Cela est dû soit au manque de temps, soit à leur faible niveau économique ou encore au fait qu'elles ne sont pas informées. 
Comparativement à certaines recherches effectuées sur l'éducation des femmes immigrées au Canada (Nkolo 2006, Statistiques Canada 2006), la nôtre prouve que notre population cible est très instruite. Les résultats de l'étude indiquent que $80 \%$ des répondantes avaient un diplôme avant leur arrivée au Canada, que $10 \%$ avaient commencé un programme universitaire, que $7 \%$ avaient un diplôme collégial et $3 \%$ un diplôme de fin d'études secondaires. Sur les $80 \%$ qui avaient obtenu un diplôme dans leur pays d'origine, $16 \%$ seulement ont obtenu une équivalence canadienne. Les autres femmes ont déclaré qu'elles n'ont pas fait la demande, justifiant cela par le fait que le système canadien ne reconnaît pas le domaine dans lequel elles ont été formées. Elles ont présumé que leur demande allait être rejetée. $8 \%$ des diplômées n'avaient pas besoin d'équivalence, leurs diplômes ont été reconnus et dès leur arrivée elles ont ainsi pu travailler dans leur domaine de formation.

Avant la migration, les femmes faisant partie de notre étude appartenaient à des sociétés traditionnelles à forte domination masculine. Leur fonction consistait à contribuer à la perpétuation ou à l'augmentation du capital social détenu par les hommes (Bourdieu). Cependant, après la migration, cette fonction change. Les femmes deviennent les principales intermédiaires dans le passage de la société traditionnelle à la société d'accueil qui est moderne. Le mode de socialisation évolue et la trajectoire socioéconomique et culturelle de la femme subit également de profondes modifications. La femme qui était exclue en tant que sujet de l'économie des échanges symboliques (Bourdieu) y joue maintenant un rôle très important. Cependant, certaines valeurs communiquées par la société d'accueil pourraient éventuellement avoir un impact sur l'intégration et le fonctionnement même des femmes africaines immigrées à Edmonton. Ces valeurs qui, pour la société d'accueil, peuvent être très importantes, paraissent parfois contradictoires aux immigrantes, selon leurs références habituelles. Quelques personnes interviewées adhèrent peu à certaines valeurs de la société d'accueil et conservent au maximum les repères du pays d'origine. Par exemple, $13 \%$ des répondantes participant à la recherche expliquent qu'elles ne se sentent pas intégrées dans les communautés d'accueil (francophone et anglophone). Non parce qu'elles sont réticentes à l'intégration, mais parce que l'intégration en soi est un processus complexe qui demande du temps. Très souvent, disent-elles, les conditions favorisant ce processus ne sont pas réunies. La répondante \# 19 a déclaré : « Si l'intégration c'est la perte de l'identité je ne serai jamais complètement intégrée, mon identité reste, il y a toujours une partie de moi qui reste au pays d'origine ». La répondante \# 15 s'est exclamée ainsi : « Je ne me sens pas vraiment Canadienne ou Albertaine ». En ce sens, les valeurs culturelles acquises sont considérées comme un marqueur identitaire. À ce moment, peut-on considérer cette façon de se comporter comme une option pour la stratégie d'évitement (Camilleri), une stratégie de sauvegarde identitaire ou du moins la recherche d'un équilibre conduisant à la survie culturelle? 


\section{Minorités visibles dans la société canadienne}

L'action sociale est associée à la structuration et aux normes établies par le système. Partant, nous pouvons mentionner que le phénomène de l'immigration a des conséquences sociopolitiques et économiques non seulement sur la vie des nouveaux arrivants, mais également sur le fonctionnement des gouvernements fédéral et provinciaux du Canada. Ces conséquences ont motivé les deux paliers gouvernementaux à créer des expressions telles que minorité visible pour catégoriser une partie de la population canadienne. Le besoin de créer une catégorie pour identifier les gens ne constitue-t-il pas un moyen de produire des différences au sein de la population?

Le concept de minorité visible est utilisé tant dans la législation canadienne que dans les statistiques officielles. Ainsi, le terme fut officiellement reconnu en 1984 et il devint l'une des quatre catégories définies dans le rapport de la Commission royale sur l'égalité dans l'emploi, conformément aux attributions de la Commission. Le concept de minorité visible est essentiellement une invention canadienne, bien qu'elle ait été utilisée, à de rares occasions, au Royaume-Uni. Elle a été intégrée à notre vocabulaire judiciaire et elle est de plus en plus utilisée dans la langue courante, explique Noël A. Kinsella (3). L'expression désigne ainsi les personnes identifiées en vertu de la Loi sur l'équité en matière d'emploi, autres que les Autochtones, qui ne sont pas de race blanche ou qui n'ont pas la peau blanche. Elle évoque des personnes dont la race ou la couleur les catégorise dans un groupe.

Statistique Canada (2006) rapporte qu'en 2006, quelque 5068100 personnes étaient membres de la population des minorités visibles, ce qui représente $16,2 \%$ de l'ensemble de la population, en hausse par rapport aux 13,4\% de l'année 2001. En Alberta, la population faisant partie du groupe de minorités visibles suit la tendance générale et s'accroît considérablement. Cependant, les immigrants venant des pays de l'Afrique francophone augmentent très rapidement : par exemple en 2001, la minorité visible constituait 5,6\% de la population francophone albertaine, soit une augmentation de plus de $18 \%$ par rapport à 1996 .

Malgré les mutations démographiques, les différences raciales dans la société canadienne se reproduisent par l'inégalité des chances et par les valeurs normatives qui résultent des faibles valeurs marchandes et sociales accordées à ceux qui ne sont pas de race blanche (Juteau 142). Étant donné que les politiques de la différence sont renforcées sur les plans économique et normatif par les expériences d'inégalité que les membres des minorités visibles affrontent dans la société canadienne, on observe ainsi un taux de chômage plus élevé chez les membres des minorités visibles que chez les autres catégories de la population, ce qui entraîne également un taux de pauvreté plus élevé dans ce groupe. Selon Clarence Lochhead et Katherine Scott : 
L'incidence de la pauvreté chez les femmes et les hommes varie en fonction de leur appartenance à une minorité visible. Ainsi, les femmes qui font partie d'une minorité visible sont plus susceptibles que les autres femmes d'avoir vécu au moins une année de pauvreté. (Lochhead et Scott 16)

La catégorisation raciale peut constituer un frein non seulement à la participation de tous à la chose publique, mais également à l'intégration qui est un processus important pour le développement de tous. La différenciation raciale affecte de façon importante la catégorie des minorités visibles et essentiellement les femmes de ce groupe au Canada. Cette différenciation a des répercussions au niveau de l'insertion économique des femmes africaines francophones qui résident à Edmonton.

Bien que les femmes jouent un rôle très important dans l'économie canadienne, il existe un grand écart, soit au niveau de l'emploi, soit au niveau de la rémunération, entre les hommes et les femmes. Les gains des femmes occupant un emploi sont souvent considérablement plus faibles que ceux des hommes. En outre, les femmes sont plus susceptibles d'avoir un emploi à temps partiel. Ce constat est vrai pour toutes les femmes : canadiennes ou immigrées. Cependant, une autre différence existe entre les femmes elles-mêmes. Pour comprendre cette réalité, il faut tenir compte de la diversité qui existe dans la société canadienne. Cette diversité peut créer plus d'obstacles qui font en sorte que les femmes immigrantes sont confrontées à deux fois plus de difficultés que les femmes nées au Canada sur le marché du travail. Les immigrantes doivent composer avec divers types de discrimination en raison de leur sexe et de leur origine ethnique. Souvent, la non-maitrise de la langue et la non-reconnaissance des titres et divers aspects de l'expérience et des compétences obtenus dans le pays d'origine constituent également d'importants obstacles à un travail rémunéré de meilleure qualité pour les immigrantes.

Les femmes immigrées, particulièrement celles des minorités visibles, connaissent des difficultés en ce qui a trait à leur insertion professionnelle. Myrlande Pierre (76) mentionne que « le racisme et le sexisme combinés créent des situations d'inégalité dans le domaine de l'emploi ». Un rapport réalisé par l'Institut canadien de recherche sur les femmes, cité par Myrlande Pierre dans son article, mentionne que « beaucoup d'employeurs jugent les femmes immigrées d'après la couleur de leur peau et entretiennent des préjugés sur leurs habitudes de travail, sur leurs aptitudes à occuper certains emplois et sur leur capacité d'intégration » (ibid. 84). Leurs compétences sont souvent remises en question par certains employeurs et collègues de travail, même lorsqu'elles occupent un poste pour lequel elles sont surqualifiées.

La majorité des femmes interrogées au cours de cette recherche a déclaré avoir choisi d'immigrer au Canada dans le but d'avoir une meilleure situation 
socioéconomique. Pour que ce changement se produise, elles ont besoin de travailler et de gagner un salaire leur permettant d'assumer leurs obligations familiales et personnelles. Sur les 30 femmes interviewées, $87 \%$ ont déclaré avoir un emploi. Pour se faire embaucher, certaines d'entre elles utilisent des réseaux de parents et d'amis, d'autres ont fait des demandes pour des postes vacants inscrits soit dans des journaux anglophones et francophones, soit sur l'Internet, soit par le biais des organismes francophones tel qu'Accès emploi qui les ont aidées dans leur recherche d'emploi.

Parmi les femmes qui sont actuellement sans emploi, $13 \%$ ont déclaré être à la recherche d'un travail, sans avoir connu de succès. Certaines de celles qui ont un emploi ont avoué qu'il ne leur a pas été facile de l'obtenir. La majorité a mis entre six mois et un an avant de se faire engager. Elles expliquent cette difficulté à trouver du travail par le fait qu'elles sont immigrantes et membres d'une minorité visible. Deux soulignent que leur origine ethnique est à la base de leurs difficultés dans la recherche d'emploi, trois pensent que c'est la couleur de leur peau qui en est la cause, neuf mentionnent la langue comme difficulté principale pour trouver du travail à Edmonton. Comme déjà mentionné, certaines femmes ont mis l'accent sur la non-reconnaissance des compétences et des acquis extérieurs, sur l'acquisition de l'expérience canadienne, sur la religion et le racisme. "Ce dernier constitue un frein à notre insertion professionnelle, car il est bel et bien présent dans la société canadienne », expliquent les femmes.

Le responsable ne va pas te dire que tu es noire, on ne peut pas t'embaucher, ce serait trop direct. Mais, il te dit des choses comme tu es surqualifiée ou tu n'as pas assez d'expérience canadienne. (Répondante \# 1)

Quand il te dit que tu es surqualifiée, tu acceptes quand même d'occuper le poste, il trouve toujours un moyen pour te refuser. Parfois, s'ils n'ont pas le choix ils t'acceptent mais ils te mènent la vie dure. (Répondante \# 2)

Si la majorité des répondantes considère la langue et le statut d'immigrante comme les principaux éléments qui ralentissent leur insertion professionnelle, pour neuf femmes, aucun de ces éléments n'a de conséquences. Le sexe, qui est toujours mentionné comme un facteur discriminatoire au niveau de l'emploi (Davis 2007, Patrimoine Canadien 1997; Statistique Canada 2001; Cardu 2002), n'a pas été mentionné par les femmes comme un obstacle dans leur recherche d'emploi.

Certaines femmes expliquent qu'il existe des discriminations au niveau salarial en lien avec leur statut d'immigrante et la durée de leur établissement au Canada. $15 \%$ déclarent que leur salaire est inférieur à celui des femmes de 
la communauté d'accueil, $15 \%$ pensent que leur salaire est inférieur à celui des femmes immigrantes qui sont arrivées au Canada avant elles, $8 \%$ croient que les hommes de la communauté d'accueil gagnent un salaire supérieur au leur et $26 \%$ disent n'avoir aucune idée de cette situation. Selon $12 \%$ des femmes, les discriminations peuvent aussi être en rapport avec l'importance du poste et le domaine dans lequel il est vacant. $12 \%$ expliquent que le salaire est égal pour ceux et celles qui ont la même compétence et qui fournissent le même travail. Cependant, $12 \%$ signalent la présence du syndicat qui empêche les employeurs d'appliquer des règles discriminatoires au niveau salarial.

À la question : à compétence égale, pensez-vous qu'il est possible d'occuper le même emploi qu'une Canadienne d'origine?, les réponses sont multiples. Neuf des répondantes pensent que oui, si les compétences et les expériences sont comparables. Quinze femmes pensent que c'est possible d'occuper le même emploi, cependant il faut maîtriser l'anglais, sinon on n'a pas les mêmes chances. "À ce moment-là nous ne pouvons pas parler de discrimination mais d'inégalité » a mentionné la répondante \# 8 . Huit femmes pensent qu'à compétence égale il n'est pas possible d'occuper le même emploi qu'une Canadienne d'origine : « il faut la dépasser en connaissance et en expérience», a relaté la répondante \#20. Quatre femmes ont déclaré qu'à compétence égale, elles doivent non seulement travailler plus qu'une Canadienne de souche, mais qu'elles doivent aussi toujours prouver leurs compétences. Selon deux femmes, la Canadienne d'origine aura la priorité au moment de l'embauche. Deux autres répondantes expliquent que la possibilité existe seulement dans le cas où l'employeur n'a pas le choix. Pour la plupart des répondantes, l'embauche d'une femme immigrante est dans plusieurs cas un choix par obligation.

Pour la majorité des femmes interviewées, une fois arrivées au Canada, leur premier souci était de trouver un emploi pour pouvoir s'occuper de leur famille. À ce moment-là, elles ne comptent plus ni sur leurs acquis et expériences antérieurs ni sur leur niveau d'éducation. Trouver un emploi devient la priorité. Trois des répondantes ont préféré retourner à l'université afin de changer de carrière. La majorité des femmes évoque la dévalorisation de leur compétence et la réticence de certains employeurs à embaucher des femmes qui ont été formées à l'extérieur. « Il y a un manque de confiance en ceux et celles qui viennent d'ailleurs », explique la répondante \# 15. « Pourquoi ne pas avoir un emploi pour lequel on a plus de compétences que celles requises?», se demande la répondante \# 30 .

Ce tableau montre le pourcentage de la population africaine occupant un emploi par rapport à l'ensemble de la population canadienne, selon le groupe d'âge et le sexe en 2001. 


\begin{tabular}{|c|c|c|c|c|c|c|}
\hline \multicolumn{4}{|c|}{ Communauté africaine } & \multicolumn{2}{c|}{ Ensemble de la population canadienne } \\
\hline Groupe d'âge & Hommes & Femmes & Total & Hommes & Femmes & Total \\
\hline 15 à 24 ans & $40,7 \%$ & $41,6 \%$ & $41,2 \%$ & $56,1 \%$ & $55,6 \%$ & $55,9 \%$ \\
\hline 25 à 44 ans & $76,0 \%$ & $62,0 \%$ & $68,9 \%$ & $85,6 \%$ & $75,2 \%$ & $80,3 \%$ \\
\hline 45 à 64 ans & $77,8 \%$ & $64,2 \%$ & $71,4 \%$ & $74,8 \%$ & $60,8 \%$ & $67,7 \%$ \\
\hline 65 ans et plus & $17,2 \%$ & $6,1 \%$ & $11,1 \%$ & $13,0 \%$ & $4,8 \%$ & $8,4 \%$ \\
\hline Total & $\mathbf{6 5 , 3} \%$ & $\mathbf{5 4 , 6} \%$ & $\mathbf{5 9 , 9} \%$ & $\mathbf{6 7 , 2} \%$ & $\mathbf{5 6 , 1} \%$ & $\mathbf{6 1 , 5} \%$ \\
\hline
\end{tabular}

Source : Statistique Canada, Recensement du Canada de 2001. Statistique Canada $\mathrm{n}^{\circ}$ 89-621-XIF au catalogue

\section{Différenciation sociale}

La différenciation sociale est considérée comme un processus économique, politique et culturel qui a des implications normatives et qui fonde les catégories sociales liées au sexe, à l'ethnicité, à la race, à l'âge et à la région d'établissement, explique Danielle Juteau (9). Elle est liée à la hiérarchisation sociale, enracinée dans l'inégalité de pouvoir, de statut, de richesse et de prestige. Elle affecte la distribution des ressources et l'intégration réelle des individus. Le concept de différenciation sociale a plusieurs significations, lesquelles ont varié avec le temps.

\section{Intersectionalité des facteurs de sexe, de classe et de race}

L'approche intersectionnelle met en relief la construction sociale des rôles et des identités. Elle cherche à identifier les rapports de pouvoir sur lesquels se basent les constructions sociales, et lutte pour mettre un terme aux rapports de domination et aux inégalités sociales. La marginalisation, la discrimination, l'exclusion et la catégorisation sont autant de facteurs qui ont poussé certains chercheurs à définir un modèle d'analyse pour examiner les effets conjoints des divers systèmes d'oppression. Ainsi, Collins (Gervais 2006) introduit le concept de « matrice des oppressions » ou encore celui de « systèmes d'oppressions entrecroisées » pour désigner l'interconnectivité entre le racisme, le sexisme et le « classisme », auxquels elle greffe d'autres grands systèmes de discrimination tels que l'hétérosexisme et le colonialisme. Le concept d'intersectionalité n'est pas ancien. Il fut proposé pour la première fois en 1991 par la juriste Kimberlé W. Crenshaw pour appréhender les législations américaines qu'elle juge inefficientes en regard des besoins exprimés par les femmes racisées victimes de violence conjugale (Corbeil et Marchand 1). Depuis, le concept apparaît comme un outil d'analyse pertinent, d'une part, pour comprendre les multiples façons (et leur répondre) dont les rapports de sexe entrent en interrelation avec d'autres aspects de l'identité sociale et, d'autre part, pour voir comment ces intersections mettent en place des expériences particulières d'oppression et de privilège. L'approche intersectionnelle insiste sur le fait que, même si les femmes blanches et les femmes noires ont une histoire commune de violence conjugale, les premières ont des privilèges que les secondes n'ont 
pas en raison du racisme et de ses effets subséquents : statut socioéconomique précaire, ghettoïsation, isolement, dévaluation professionnelle (Gervais et al. 2006). Angela Davis utilise l'intersectionalité pour analyser la situation des femmes qui, selon elle, est reliée non seulement au sexe, mais aussi à la race et à la classe. Elle relate que le sort des femmes, spécifiquement celui des femmes noires, résulte des déséquilibres provoqués par les hiérarchies établies. (Davis 32)

La différenciation sociale se développe à partir de variables multiples et diverses, s'agençant de telle façon à réglementer la vie sociale. Ainsi, la différence sociale engendre la constitution de groupes sociaux différents basés sur des traits comme le sexe, l'appartenance sociale et l'origine ethnique. Certaines femmes immigrantes africaines francophones d'Edmonton vivent ces différences. Arrivent-elles à les dépasser pour s'intégrer dans la société d'accueil, ou du moins, ces différences constituent-elles des barrières freinant leur intégration? Au Canada, peut-on considérer l'égalité des sexes comme un acquis ou existe-t-il encore certaines différences liées non seulement au sexe, mais aussi à l'appartenance sociale et ethnique?

\section{Intégration}

Du point de vue positif, l'intégration est la participation totale et active à la vie de la nouvelle société dont l'immigrant devient un membre à part entière. C'est également être reconnu par les autres membres de la communauté nationale comme faisant partie d'elle. C'est aussi la possibilité d'accéder aux divers services, prestations et moyens que cette société met à la disposition de chacun de ses membres, explique Jean-Claude Ricci (Resch 13). Cependant, quand l'intégration prend le sens de l'assimilation, elle devient négative. Jean-Claude Ricci explique que " l'intégration est relative à l'accueil de l'Autre, au débat sur le rôle de l'étranger, sur sa place dans la cité » (ibid). C'est un exercice délicat et difficile, car intégrer est une attitude nécessaire qui provoque parfois de grandes réticences, soit de la part de la communauté d'accueil, soit de la part de l'immigrant lui-même. Dans le cas de la communauté d'accueil, les principales causes de cette réticence se basent principalement sur la crainte d'être envahie par les étrangers, la peur de perdre son identité, enfin la méconnaissance de l'autre. Dans le cas des immigrants, cette réserve repose surtout sur la peur d'être rejetés ou incompris, sur le stéréotype et sur le manque d'informations relatives à la communauté d'accueil.

\section{Femmes immigrantes et processus d'intégration socioéconomique}

Les femmes tiennent un rôle central dans le dynamisme d'intégration des membres de la famille à la société. Face aux différences déjà mentionnées, comment s'y prennent-elles pour jouer ce rôle? Des études ont souligné que les immigrantes doivent composer avec de multiples niveaux de discrimination en raison de leur sexe et de leur origine ethnique. Ce constat semble s'appliquer 
aux femmes immigrées sans distinction de profil. Souvent, le processus menant à leur intégration est beaucoup plus lent que pour les autres membres de leur famille, car elles privilégient leurs enfants et l'insertion socioprofessionnelle de leur conjoint avant de commencer leurs propres recherches d'emploi, par exemple. En mettant l'accent sur leur rôle de mère, elles accusent un retard non seulement dans leur intégration sociale, mais aussi dans leur progression de carrière, souligne Hélène Cardu (87). L'immigration entraîne souvent une rupture radicale par rapport aux habitudes de vie, ce qui peut avoir une influence importante sur l'intégration sociale et professionnelle des femmes. Une redéfinition des rôles de chaque membre de la famille s'impose. Les femmes peuvent connaître des phases d'isolement dans leur adaptation socioprofessionnelle, car elles doivent adopter des statuts nouveaux et composer avec la dévalorisation de statuts anciens, par exemple le rôle de mère à la maison (ibid. 87). Une telle situation peut engendrer des cassures au niveau de leur trajectoire socioprofessionnelle. Dans ce cas, Hélène Cardu souligne l'importance de la présence des réseaux de soutien familiaux, ethniques et communautaires. De nombreuses études, telles que «Les églises chrétiennes africaines et l'intégration sociale/citoyenne des nouveaux arrivants africains dans la région d'Edmonton » (Mulatris, 2009) montrent qu'au début de la transition socioculturelle, il y a souvent un repli sur la communauté ethnique d'origine, sur l'identité ethnoculturelle, et que les réseaux de soutien ethnoculturels deviennent d'une importance primordiale. La perte des références habituelles, à laquelle s'ajoutent d'autres difficultés telles que la discrimination, le stéréotype, l'exclusion et le sentiment de perdre le contrôle de soi peuvent rendre encore plus difficile le processus d'intégration chez les femmes.

Le phénomène d'intégration a, selon les répondantes, différentes significations. L'intégration d'un immigrant, nous le savons, est la combinaison des efforts de plusieurs acteurs : l'immigré lui-même et les gens de la société qui l'accueille. En même temps, l'intégration relève d'abord de la perception que l'immigrant a de lui-même, dans son nouveau cadre de vie. Il s'agit d'un parcours individuel certes, mais qui nécessite l'appui et le soutien d'autres intervenants. Dans le cas des femmes immigrantes originaires de l'Afrique noire par exemple, les éléments qui sont considérés comme des obstacles par certaines ne le sont pas chez d'autres. En leur demandant de noter les éléments faisant obstacle à leur intégration, les femmes immigrantes ont donné des réponses multiples. Certains éléments considérés au préalable comme ayant une grande influence sur le processus d'intégration des femmes africaines francophones immigrées à Edmonton ne sont pas cités parmi les obstacles prioritaires.

Onze femmes ont affirmé avoir subi le racisme à cause de leurs noms, ce qui a certainement une influence dans le processus de leur intégration. La présence des enfants en bas âge est, pour certaines, considérée comme un obstacle. Car, en plus de travailler parfois à temps plein, ce sont elles qui doivent s'occuper des enfants, ce qui leur laisse peu de temps pour des activités 
de socialisation par exemple. Ici, ne pouvons-nous pas percevoir un reflet de la division sexuelle du travail? Certaines pensent que la couleur de leur peau est un obstacle, « car même si les gens ne l'expriment pas ouvertement on le sent », a relaté l'une des participantes au focus group.

\section{Communauté ethnique et femmes africaines francophones immigrées à Edmonton}

Dans la majorité des cas, la communauté ethnique n'a joué aucun rôle dans l'intégration des femmes. $60 \%$ déclarent qu'elles n'ont eu aucun apport de leur communauté en arrivant ici. Certaines expliquent qu'elles faisaient partie des premières arrivées de leurs communautés. Selon d'autres, la communauté ethnique accorde plus d'importance au maintien des liens avec le pays d'origine et à la transmission des valeurs culturelles aux jeunes générations qu'à l'accompagnement les nouveaux arrivants dans leur intégration à la société d'accueil. Cependant, $40 \%$ des participantes mentionnent que leurs communautés ethniques ont joué un rôle très important en ce qui concerne leur intégration. Elles ont obtenu des informations sur les services et les institutions existants et ont reçu du support sur les plans psychologique, moral, social et parfois financier. La plupart des femmes considère les rencontres d'échange organisées par les communautés ethniques comme des éléments importants dans le processus d'intégration. Ces rencontres permettent de côtoyer des personnes d'autres cultures, activités jugées utiles par certaines femmes.

\section{Contribution au développement social et économique d'Edmonton}

Le développement socioéconomique entraîne généralement une progression du niveau de vie de la population. Sur le plan économique, le développement désigne des croissances positives qui occasionnent la création de richesses et l'amélioration des conditions de vie. Généralement, le développement économique et social fait référence à l'ensemble de mutations que peut connaître une zone géographique par exemple. Pour mesurer cette progression, outre les indicateurs traditionnellement utilisés, tels que le produit intérieur brut (PIB) et le produit national brut (PNB), d'autres indicateurs sont également considérés comme l'indice de développement humain (IDH) où sont associées l'éducation et la santé, pour ne citer que ces deux éléments. Dans cet article, le concept de développement socioéconomique fait surtout référence à l'intégration des femmes africaines noires francophones dans leur communauté d'accueil, tant sur le plan économique - en ayant un emploi stable qui constitue un élément important dans leur processus d'intégration et qui est essentiel à leur participation et à leur contribution dans la société d'accueil; que sur le plan social - en ayant des connaissances sur les institutions existantes, sur la langue et sur l'histoire de cette société. Ces éléments sont des facteurs importants qui peuvent conduire à une intégration réussie.

Généralement, on considère l'apport des immigrants à leur société d'accueil au niveau de l'accroissement démographique, de la main d'œuvre et de la diversité 
culturelle. Cependant, en se basant sur le niveau de scolarité des immigrants, on ne peut pas nier l'apport de leur capital humain au développement de leur communauté d'accueil. On peut donc ainsi considérer l'immigration comme un apport important au dynamisme même de la société d'accueil. En ce qui concerne les femmes africaines francophones faisant partie de notre recherche, la majorité contribue non seulement à la diversité et à l'innovation de la société d'accueil, elle participe également à sa croissance économique.

Les femmes interrogées perçoivent leur contribution au développement social et économique de la région d'Edmonton de différentes façons. Sur le plan social, $67 \%$ des répondantes disent qu'elles contribuent au développement d'Edmonton. Les raisons évoquées sont la réalisation des activités sociocommunautaires et religieuses, la participation active dans la société, le bénévolat, la participation aux activités avec les enfants, l'aide apportée aux nouveaux arrivants pendant leur première installation, la socialisation et le dynamisme. D'après elles, ces différents apports montrent qu'elles participent pleinement à la vie sociale d'Edmonton, ce qui leur permet de dire qu'elles contribuent sûrement au développement social de la région. En revanche, $33 \%$ affirment ne pas contribuer au développement social d'Edmonton, soit par le fait qu'elles ne connaissent pas assez la communauté, soit à cause de la langue. «Comment contribuer au développement quand on n'arrive même pas à s'exprimer dans la langue de la majorité? », se demande la participante \# 3 .

Sur le plan économique, la majorité des femmes interviewées, soit $80 \%$ d'entre elles, déclarent apporter leur contribution. Pour illustrer leur participation, elles révèlent différents éléments concernant la question. Par exemple, elles rappellent qu'elles paient des taxes et soulignent le fait qu'elles ont un emploi et qu'elles sont des consommatrices. Cependant, $13 \%$ des femmes estiment qu'elles ne participent pas au développement économique d'Edmonton parce qu'elles ne travaillent pas, mais, ajoutent-elles : « Nous consommons et nous payons également des taxes ».

L'intégration est le phénomène le plus discuté et le plus commenté par les répondantes. Complexe, le processus d'intégration est lent pour certaines femmes. De plus, nombre d'éléments sont à considérer dans ce processus. La seule volonté ne suffit pas, bien qu'elle soit très importante. Parmi les personnes interrogées, celles qui ont pu compter sur des réseaux à leur arrivée ont eu moins de difficultés dans leur processus d'intégration. Elles ont obtenu de l'aide non seulement sur les plans social et économique, mais aussi psychologique et moral, ce qui a favorisé leur démarche. La majorité des répondantes se disent intégrées tant sur le plan social qu'économique et estiment apporter leur contribution au développement de la capitale albertaine. 


\section{Perspective critique}

Les phénomènes étudiés dans cet article nous ont permis de dévoiler la dure réalité dans laquelle évoluent les femmes africaines immigrantes, particulièrement les francophones vivant à Edmonton. L'installation dans une nouvelle société, où tout est totalement différent, provoque souvent des inquiétudes qui proviennent des écarts existant entre le mode de vie connu au pays d'origine et celui auquel les femmes doivent s'adapter au pays d'accueil. Ainsi le projet de l'immigrante peut prendre une forme différente de celle planifiée avant le départ. Le plus souvent, les idéaux de vie seront grandement modifiés au contact de la nouvelle réalité. La façon dont l'immigration est perçue, comprise et vécue par la femme immigrée peut constituer un atout majeur dans son processus d'intégration et d'inclusion. Généralement partagée entre des champs socioculturels différents, l'immigrante peut faire face non seulement à des conflits internes, mais également à la discrimination et aux différenciations sociales.

Les femmes faisant partie de notre étude ont connu certaines formes de différenciation et de discrimination sociales inexistantes dans leur pays d'origine. Celles-ci agissent fortement sur leurs attentes. Au cours des focus group et des entrevues réalisés avec certaines femmes africaines francophones qui immigrent à Edmonton, nous avons pu ressentir un niveau de frustration, d'amertume, de désillusion et parfois même de résignation. Pour la plupart d'entre elles, la volonté de recommencer une nouvelle vie est forte, mais pas suffisante. Des mesures actives d'accompagnement sont nécessaires pour les aider dans leur cheminement, et surtout les aider à reprendre confiance en elles. Certaines de nos répondantes ont dénoncé les discriminations subies surtout au niveau de leur recherche d'emploi. Certaines limitations, qui ne sont pas particulières aux femmes africaines, comme la langue, la religion et l'ethnicité, ne font qu'accentuer ces formes de discrimination et de différenciation.

Bien que le Canada soit connu comme un pays multiculturel et que sur le plan constitutionnel (Loi sur le multiculturalisme, 1988), certaines mesures aient été prises pour contrecarrer certains types de différences sociales, de racisme et de discrimination, celles-ci n'empêchent pas que les immigrants, spécifiquement les femmes, subissent certains types de discrimination et de racisme. Ces mesures sont-elles suffisantes, ou du moins sont-elles appliquées de façon à générer les résultats espérés? Les réponses données par les femmes interviewées prouvent qu'il y a beaucoup à faire dans ce domaine, surtout sur le plan socioéconomique, pour qu'elles puissent se sentir moins discriminées et entrevoir plus d'ouverture dans leur démarche vers une intégration réussie. Des actions plus visibles et plus actives doivent être mises en place pour encourager leur participation et leur implication, dans le but de construire une société plus tolérante et plus juste où tout le monde aura sa place. 
Cependant, les immigrantes africaines francophones ont également leur part de responsabilité dans ce processus. Pour se faire une place dans la société d'accueil, elles méritent certainement d'avoir de meilleures conditions d'intégration provenant des instances concernées, mais elles doivent aussi faire des efforts pour rejoindre l'autre, pour s'inclure et s'intégrer, pour partager et comprendre la nouvelle réalité dans laquelle elles sont appelées à évoluer. Ce sont des moyens indispensables à tout processus d'intégration et d'épanouissement.

\section{Conclusion}

Cette étude porte sur les femmes africaines francophones installées à Edmonton depuis au moins cinq ans. Au cours de cette recherche, nous avons analysé certains éléments qui encouragent ou ralentissent leur processus d'intégration. Nous avons exploré leur niveau d'éducation, leur formation professionnelle et leurs expériences de travail à Edmonton et aux pays d'origine. Au cours des entretiens et des focus group réalisés, nous avons eu l'occasion de mettre en évidence des éléments favorables à l'intégration, et d'autres qui sont considérés comme des barrières auxquelles les femmes font face dans leur effort pour arriver à une intégration socioéconomique réussie. La majorité de nos répondantes possèdent un niveau élevé d'éducation et occupent un emploi à temps plein, même si parfois l'emploi occupé n'honore pas leur formation, leurs compétences et leurs expériences antérieures. Cela est généralement dû à la non-reconnaissance des diplômes et des expériences acquis à l'extérieur du Canada, au manque d'expérience canadienne, à certains préjugés liés à la race et au statut d'immigrant et au fait qu'elles ne maitrisent pas l'anglais.

L'apport au développement socioéconomique varie en fonction de certaines différenciations sociales et discriminations qui affectent une partie des femmes immigrantes de notre étude. Si le sexe a toujours été considéré comme un facteur de discrimination en ce qui a trait à l'emploi et à la rémunération, les répondantes ont affiché une attitude contraire au cours de cette recherche. Aucune d'elles n'a mentionné le sexe comme une entrave dans leur recherche d'emploi ou un facteur pouvant influencer leur salaire. Le sexe ne peut donc pas être considéré comme l'un des critères socioculturels de discrimination et de différenciation. Par contre, le fait que la présence des enfants en bas âge soit considérée comme un obstacle à l'intégration de certaines femmes invite à penser ce problème d'un point de vue culturel. Cette question mérite d'être approfondie par des études plus exhaustives pouvant s'étendre à une population plus large. Pour certaines femmes, en revanche, la question raciale représente encore aujourd'hui un indice de discrimination et d'exclusion au niveau de l'emploi. L'intégration socioéconomique des femmes immigrantes africaines francophones n'est pas simplement une question de choix personnel : ce choix doit s'inscrire dans des conditions favorisant le processus d'intégration en question. 


\section{Bibliographie}

Assemblée générale des Nations unies. Migrations internationales et développement. 2006. Consulté le 19 février 2009. http://daccessdds.un.org/doc/UNDOC/GEN/ N06/353/55/PDF/N0635355.pdf? OpenElement

Blanc-Chaleard, M.C. Histoire de l'immigration. Paris : Éditions La Découverte, 2001. Bourdieu, P.. Raisons pratiques sur la théorie de l'action. Paris : Éditions du Seuil, 1994.

Bourdon, R., et F. Bourricaud. Dictionnaire critique de la sociologie. Paris : PUF, $3^{\mathrm{e}}$ édition, 2004.

Cardu, H., et M. Sanschagrin. « Les femmes et la migration : les représentations identitaires et les stratégies devant les obstacles à l'insertion socioprofessionnelle à Québec » Recherche féministe 15.2 (2002) : 87-122.

Camilleri, C., et al. Stratégies identitaires. Paris : PUF, 1990.

Cazeneuve, J. Dix grandes notions de sociologie. Paris : Éditions du Seuil, 1976.

Centre d'actualité de l'ONU, HCR. Le XIXe siècle est celui du déplacement humain. 2007. Consulté le 23 février 2009. http://www.un.org/apps/newsFr/storyF.asp? News $I D=14905 \& C r=H C R \& C r 1=$ migrations

Centre d'études ethniques de l'Université de Montréal. Actes du séminaire sur les indicateurs d'intégration des immigrants, Québec, 1994.

Coenen-Huther, J. Le fonctionnalisme en sociologie : et après? Bruxelles : Éditions de l'Université de Bruxelles, 1984.

Commission de la fonction publique. Conférence sur les minorités visibles dans la Fonction publique fédérale (Région de l'Atlantique), 2006. Consulté le 12 mars 2009. http://www.psc.cfp.gc.ca/spch-disc/2006/2006-02-20-fra.htm

Condition féminine du Canada. Intégration et immigration tenant compte des rapports sociaux entre les sexes. 1987-1996.

Conseil national des minorités visibles de la fonction publique. Le rapport sur l'équité d'emploi. 2006. Consulté le 12 avril 2009. http://ncvm.gc.ca/index.php/ fr/library/eereport

Corbeil, C., et I. Marchand. Penser l'intervention féministe à l'aune de l'approche intersectionnelle. Défis et enjeux pour l'intervention auprès des femmes marginalisées. 2006. Consulté le 10 mars 2009. http://www.relaisfemmes. qc.ca/files/annexes_Corbeil_Marchand.pdf

Couture, C., et J. Bergeron. L'Alberta et le multiculturalisme francophone : témoignages et problématique. Centre d'études canadiennes, Faculté St Jean, Université de l'Alberta, 2002.

Cuche, D. La notion de culture dans les sciences sociales. Paris : Éditions La Découverte, 1996.

Davis, A. Femmes, race et classe. Paris : Des Femmes Antoinette Fougue, 2e édition, 2007.

Dubé, P. Immigration, langue et identité dans l'Ouest canadien. 2004. Consulté le 05 février 2009. http://atlanticportal.hil.unb.ca:8000/archive/00000084/02/ Dube_French.pdf

Gervais, L., et al. « Penser l'intervention féministe à l'aune de l'approche intersectionnelle, défis et enjeux » Érudit 19.1 (2006) : 40-57.

Frenette, M., et R. Morisette. Convergeront-ils un jour? Les gains des travailleurs immigrants et de ceux nés au Canada au cours des deux dernières décennies. Catalogue no. 11F0019MIF - No. 215. Ottawa : Statistique Canada, 2006. 
Girard, M., et al. « Intégration économique des nouveaux immigrants : Adéquation entre l'emploi occupé avant l'arrivée au Québec et les emplois occupés depuis l'immigration » Cahiers Canadiens de Sociologie 33.4 (2008) : 791-814.

Henripin, J. La Métamorphose de la population canadienne. Montréal : Éditions Varia, 2003.

Institut canadien de recherche sur les femmes. Femmes et pauvreté : vivre le racisme au féminin. 2002. Consulté le 12 mars 2009. http://www.criaw-icref.cal indexFramef.htm

Juteau, D., (dir.). La différenciation sociale : modèles et processus. Montréal : PUM, 2003.

Kinsella, N. Les Nations Unies et l'expression "minorité visible » : l'incidence de l'hypothèse de Whorf sur les droits de la personne. Université de Regina, Conférence GLOBUS, 2007. Consulté le 6 avril 2008. http://www.sen.parl. gc.ca/nkinsella/PDF/Speeches/SoberSecondThought-f.pdf

Kymlicka, W. La voie Canadienne, repenser le multiculturalisme. Montréal : Éditions Boréal, 2003.

Lamotte, A. «Femmes immigrées et reproductions sociales » Recherches sociographiques 32.3 (1991) : 367-384.

Lallement, M. Histoire des idées sociologiques de Parsons aux contemporains. Paris : Armand Collin, 3e édition, 2007.

Li, P. "Earning Disparities Between Immigrants and Native-born Canadians" Canadian Review of Sociology and Anthropology 37.3 (2000) : 289-311.

-. "The Market Worth of Immigrants Educational Credential" Canada Public Policy 27.1 (2001) : 23-38.

—. "Initial Earnings and Catch-up Capacity of Immigrants" Canadian Public Policy 29.3 (2003) : 319-337.

Lochhead, C., et Scott K. La dynamique de la pauvreté chez les femmes au Canada. Ottawa : Condition féminine Canada, 2000.

Mulatris, P. « L'immigration francophone africaine et noire en Alberta : Chemins parcouru en 20 ans » Le Franco 43.9 (27 février 2009) : pages.

-. «Enquête sur l'immigration francophone en Alberta ». Association canadienne française de l'Alberta, 2008.

—. «Francophonie albertaine et inclusion des nouveaux arrivants : post mortem à un débat sur un changement de nom » Revue de l'intégration et de la migration internationale 10.2 (2009) : pages.

Nkolo, C. «Processus d'insertion professionnelle de femmes immigrantes "noires" d'origine africaine détentrices d'un grade universitaire et résidentes de la ville de Québec ». Thèse de doctorat, Département, Université..., 2008.

Pélissier, M., et M. Paecht. Les modèles d'intégration en questions. Enjeux et perspectives. Paris : PUF, 2004.

Pierre, M. « Les facteurs d'exclusion faisant obstacle à l'intégration socioéconomique de certains groupes de femmes immigrées au Québec. Un état des lieux » Nouvelles pratiques sociales 17.2 (2005) : 75-94.

Piaget, J. Le structuralisme. Paris : PUF, 7e édition , 1979.

Quivy, R., et V. Campenhoudt. Manuel de recherche en sciences sociales. Paris : Dunod, $3^{\mathrm{e}}$ édition, 2006.

Recherche du conseil du statut de la femme. Les femmes immigrées du Québec: Quelques éléments d'un portrait socio-économique, 1993. 
Resch, Y. Définir l'intégration? Perspectives nationales et représentations symboliques. Montréal : XYZ, 2001.

Rocher, G. Talcott Parsons et la sociologie américaine. Paris : PUF, 1 ère édition, 1972.

Statistique Canada. Le Quotidien, Recensement de 2006 : immigration, citoyenneté, langue, mobilité et migration, 2007. \# d'enquête 3901. Consulté le 24 février 2009. http://www.statcan.gc.ca/daily-quotidien/071204/dq071204a-fra.htm

Statistique Canada. Recensement de la population de 2006. Produit $n^{\circ}$ 97-557XCB2006021 au catalogue de Statistique Canada, 2006.

Statistique Canada. Edmonton, Alberta (code 4811061). Profil des communautés de 2006. Produit no 92-591-XWF au catalogue de Statistique Canada, 2007.

Statistique Canada. Recensement 2006 : Origine ethnique et minorités visibles. 2008. Consulté le 11 mars 2009. http://www12.statcan.ca/census-recensement/ 2006/rt-td/eth-fra.cfm.

Schnapper, D. La relation à l'Autre. Au cour de la pensée sociologique. Paris : Gallimard, 1998.

Talcott, P. The social system. Glencoe, IL: Free Press, 1951.

Tremblay, A. Sondage : Histoire, Pratique et Analyse, Québec: Université Laurentienne, 1991. 